\title{
Mechanical Failure of Transparent Flexible Silver Nanowire Networks for Solar Cells using 3D X-Ray Nano Tomography and Electron Microscopy
}

\author{
$\underline{\text { Silvan Englisch }}^{1 *}$, Janis Wirth ${ }^{1}$, Nadine Schrenker ${ }^{1}$, Kai Cheong Tam ${ }^{2}$, Hans-Joachim Egelhaaf ${ }^{2}$, \\ Christoph J. Brabec ${ }^{2}$, Erdmann Spiecker ${ }^{1}$. \\ 1. Institute of Micro-and Nanostructure Research (IMN) \& Center for Nanoanalysis and Electron \\ Microscopy (CENEM), Friedrich-Alexander University, Erlangen, Germany \\ 2. ZAE Bayern: Bavarian Center for Applied Energy Research, Erlangen, Germany \\ *Corresponding author, silvan.englisch@fau.de
}

For applications as flexible organic solar cells (OSCs) or "smart clothing", which means clothing with integrated electronics it is decisive that the active layers as well as the electrodes withstand mechanical loading. Indium tin oxide (ITO), which is still a common material as electrode, behaves brittle under deformation and is relatively expensive. Metallic nanowire networks, especially silver nanowires (AgNWs), are highly promising alternatives [1]. They fulfill the requirement of a low sheet resistance combined with high transmittance. On a macroscopic scale bending tests as well as tensile tests revealed the excellent performance of $\mathrm{AgNW}$ films, since the increase of resistance is small compared to ITO films [2].

In this contribution, the authors report 3-dimensional characterization of OSCs with AgNW electrodes. The sample exhibit significant regions of interest at the nanoscale of $70 \mathrm{~nm}$ to $170 \mathrm{~nm}$. At the University of Erlangen-Nürnberg a high-resolution XRM/NanoCT instrument (ZEISS Xradia 810 Ultra) has been recently installed and put into operation. The instrument uses Fresnel zone plate optics to achieve 3D resolutions down to $50 \mathrm{~nm}$ and can be equipped with a Zernike phase ring enabling phase contrast in addition to standard absorption contrast imaging. Therefore, high resolution Scanning Electron Microscopy (SEM) and Scanning Transmission Electron Microscopy (STEM) can be nicely combined with XRM to image the network either as 2D labour sample or buried between layers as the complete solar cell device (Fig. 1, 3). Scale-bridging analysis across multiple microscopy techniques, analytical electron microscopy and XRM as well as NanoCT is required for gaining deeper insight into the structure. However, the material system features regions of interest at the optical resolution limit, which exploit the potential of labour XRM. In combination with tomographic reconstruction non-destructive 3D characterization can be performed.

In order to understand failure mechanisms and prospectively optimize the deformation behaviour of AgNW electrodes we perform micromechanical tests of these networks as well as of complete solar cell devices. Figure 2a) shows a 2D Network of AgNWs with a diameter of $90 \mathrm{~nm}$ after straining up to $30 \%$ in the SEM. The images reveal the connectivity and, therefore, the conductivity of the AgNW networks. By combining absorption and phase contrast imaging in XRM the nanonetwork and crack-distribution can be independently revealed, even if buried in different layers when used in a complete solar cell device (Fig. 2b)). In combination with NanoCT this enables non-destructive characterization of the function status of the working organic optoelectronic device. 
Currently, we perform interupted tensile test for SEM as well as focus on in situ micromechanical tests for SEM and XRM.

\section{References:}

[1] Guo, F. et al., Energy Environ. Sci. 8, (2015).

[2] Nickel, F. et al., Sol. Energy Mater. Sol. Cells 130, 317-321 (2014).

[3] The authors thank the DFG for financial support within the frameworks of the research training group GRK1896 "In situ Microscopy with Electrons, X-rays and Scanning Probes" and the project SP648/8 "Hochauflösende Röntgenmikroskopie für korrelative Tomographie, Hochdurchsatzscreening und in situ mechanische Prüfung von Struktur- und Funktionswerkstoffen"
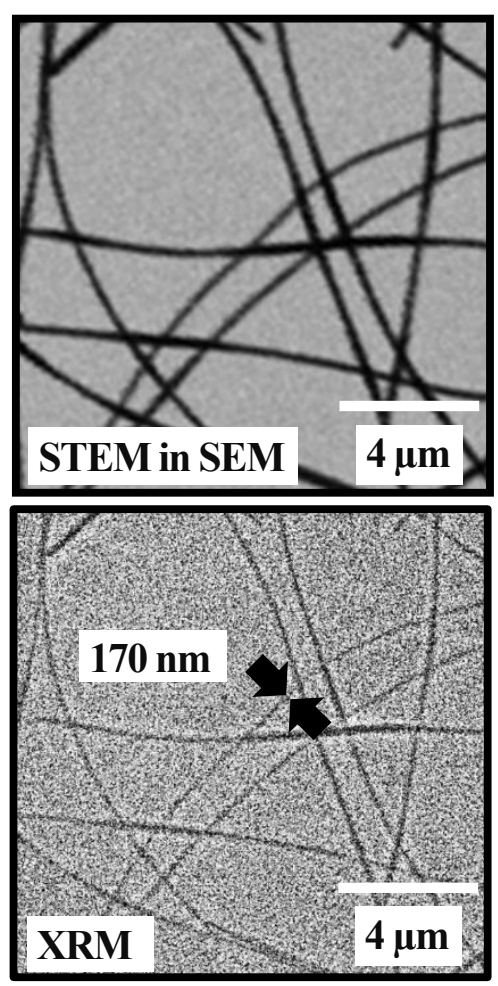

Figure. 1. a) STEM and b) XRM image of AgNW with a diameter of 170 nm. Same area shown. Figure b) provided Steve Kelly ZEISS Ag.
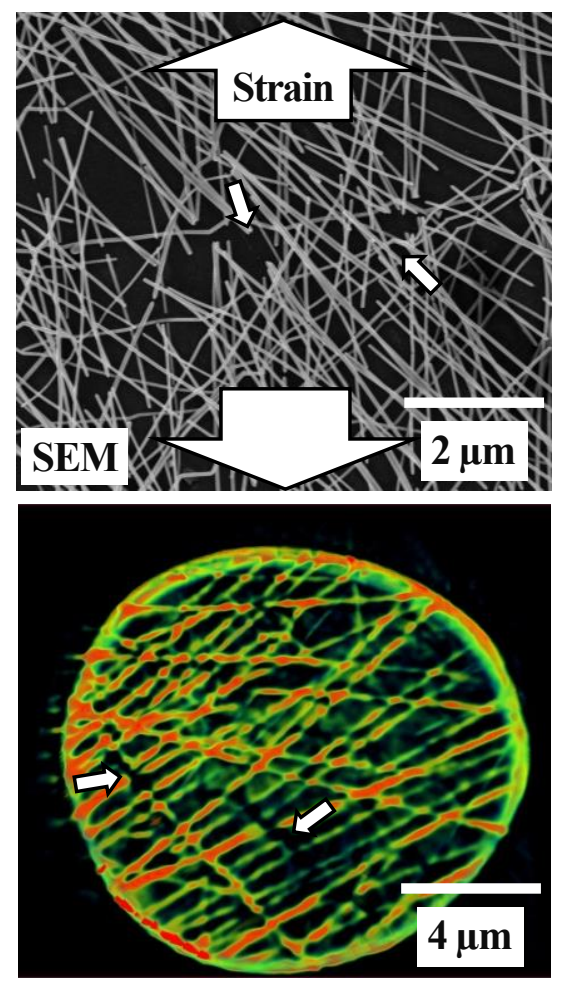

Figure. 2. a) SEM image of up $30 \%$ strained top layer $\mathrm{AgNW}$ with a diameter of $90 \mathrm{~nm}$ of a complete solar cell. b) XRM reconstruction of the buried bottom layer of the solar cell. Crack distribution shown in both layers.

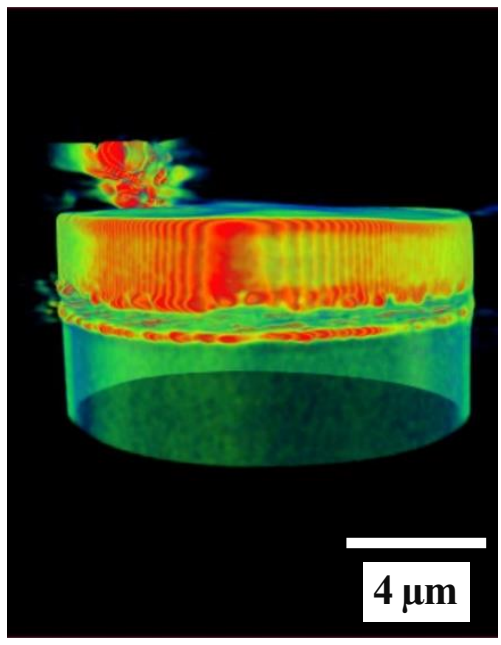

Figure. 3. XRM reconstruction of multilayered solar cell from side tomography. Both buried networks observed. 